\title{
FIXED POINT SETS OF MAPS HOMOTOPIC TO A GIVEN MAP
}

\author{
CHRISTINA L. SODERLUND
}

Received 3 December 2004; Revised 20 April 2005; Accepted 24 July 2005

Let $f: X \rightarrow X$ be a self-map of a compact, connected polyhedron and $\Phi \subseteq X$ a closed subset. We examine necessary and sufficient conditions for realizing $\Phi$ as the fixed point set of a map homotopic to $f$. For the case where $\Phi$ is a subpolyhedron, two necessary conditions were presented by Schirmer in 1990 and were proven sufficient under appropriate additional hypotheses. We will show that the same conditions remain sufficient when $\Phi$ is only assumed to be a locally contractible subset of $X$. The relative form of the realization problem has also been solved for $\Phi$ a subpolyhedron of $X$. We also extend these results to the case where $\Phi$ is a locally contractible subset.

Copyright (c) 2006 Christina L. Soderlund. This is an open access article distributed under the Creative Commons Attribution License, which permits unrestricted use, distribution, and reproduction in any medium, provided the original work is properly cited.

\section{Introduction}

Let $f: X \rightarrow X$ be a self-map of a compact, connected polyhedron. For any map $g$, denote the fixed point set of $g$ as Fix $g=\{x \in X \mid g(x)=x\}$. In this paper, we are concerned with the realization of an arbitrary closed subset $\Phi \subseteq X$ as the fixed point set of a map $g$ homotopic to $f$.

Several necessary conditions for this problem are well known. If $\Phi=$ Fix $g$ for some map $g$ homotopic to $f$, it is clear that $\Phi$ must be closed. Further, by the definition of a fixed point class (cf. [1, page 86], [7, page 5]), all points in a given component of $\Phi$ must lie in the same fixed point class. Thus, as the Nielsen number (cf. [1, page 87], [7, page 17]) of any map cannot exceed the number of fixed point classes and as the Nielsen number is also a homotopy invariant, the set $\Phi$ must have at least $N(f)$ components. In particular, if $N(f)>0$ then $\Phi$ must be nonempty. It is also necessary that $\left.f\right|_{\Phi}$, the restriction of $f$ to the set $\Phi$, must be homotopic to the inclusion map $i: \Phi \hookrightarrow X$.

In [12], Strantzalos claimed that the above conditions are sufficient if $X$ is a compact, connected topological manifold with dimension $\neq 2,4$, or 5 and if $\Phi$ is a closed nonempty subset lying in the interior of $X$ with $\pi_{1}(X, X-\Phi)=0$. However, Schirmer disproved this claim in [10] with a counterexample and presented her own conditions, (C1) and (C2). 
2 Fixed point sets of maps homotopic to a given map

Definition 1.1 [10, page 155]. Let $f: X \rightarrow X$ be a self-map of a compact, connected polyhedron. The map $f$ satisfies conditions (C1) and (C2) for a subset $\Phi \subseteq X$ if the following are satisfied (the symbol $\backsim$ denotes homotopy of paths with endpoints fixed and $*$ the path product):

(C1) there exists a homotopy $H_{\Phi}: \Phi \times I \rightarrow X$ from $\left.f\right|_{\Phi}$ to the inclusion $i: \Phi \hookrightarrow X$,

(C2) for every essential fixed point class $\mathbb{F}$ of $f: X \rightarrow X$ there exists a path $\alpha: I \rightarrow X$ with $\alpha(0) \in \mathbb{F}, \alpha(1) \in \Phi$, and

$$
\{\alpha(t)\} \backsim\{f \circ \alpha(t)\} *\left\{H_{\Phi}(\alpha(1), t)\right\} .
$$

The latter condition, (C2), reflects Strantzalos' error. He apparently overlooked the $H$-relation of essential fixed point classes of two homotopic maps (cf. [1, pages 87-92], [7, pages 9, 19]).

Schirmer showed that (C1) and (C2) are both necessary conditions for realizing $\Phi$ as the fixed point set of any map $g$ homotopic to $f$ ([10, Theorem 2.1]). She then invoked the notion of by-passing ([9, Definition 5.1]) to prove the following sufficiency theorem. A local cutpoint is any point $x \in X$ that has a connected neighborhood $U$ so that $U-\{x\}$ is not connected.

TheOREM 1.2 [10]. Let $f: X \rightarrow X$ be a self-map of a compact, connected polyhedron without a local cutpoint and let $\Phi$ be a closed subset of $X$. Assume that there exists a subpolyhedron $K$ of $X$ such that $\Phi \subset K$, every component of $K$ intersects $\Phi, X-K$ is not a 2-manifold, and $K$ can be by-passed. If (C1) and (C2) hold for $K$, then there exists a map g homotopic to $f$ with Fix $g=\Phi$.

Observe that Schirmer's theorem permits $\Phi$ to be any type of subset, provided it lies within an appropriate polyhedron $K$. However, all the required conditions are placed on the polyhedron $K$. If we wish to prove that $\Phi$ can be the fixed point set, then we should require that our conditions be on $\Phi$ itself. We can remedy this problem with a statement equivalent to that of Theorem 1.2.

THeOREM 1.3. Let $f: X \rightarrow X$ be a self-map of a compact connected polyhedron without a local cutpoint and let $\Phi$ be a closed subpolyhedron of $X$ satisfying

(1) $X-\Phi$ is not a 2-manifold,

(2) (C1) and (C2) hold for $\Phi$,

(3) $\Phi$ can be by-passed.

Then for every closed subset $\Gamma$ of $\Phi$ that has nonempty intersection with every component of $\Phi$, there exists a map $g$ homotopic to $f$ with Fix $g=\Gamma$. In particular, if $\Phi$ is connected, then every closed subset of $\Phi$ (including $\Phi$ itself) is the fixed point set of a map homotopic to $f$.

Although Theorem 1.3 requires $\Phi$ to be a subpolyhedron, the subset $\Gamma \subseteq \Phi$ is subject to few restrictions, thus preserving the broad scope of Schirmer's original theorem.

In Section 3 we extend Theorem 1.3 to the case where $\Phi$ is a closed, locally contractible subset of $X$, but not necessarily a polyhedron. The result is given in Theorem 3.5. Since the class of closed, locally contractible spaces contains the class of compact, connected 
polyhedra, this extension broadens the scope of the sufficiency theorem. Moreover, polyhedral structure is a global property, whereas local contractibility is a local property and thus presumably easier to verify.

We examine a similar question for maps of pairs in Section 4. For any map $f:(X, A) \rightarrow$ $(X, A)$ of a polyhedral pair, $\mathrm{Ng}([8])$ presented necessary and sufficient conditions for realizing a subpolyhedron $\Phi \subseteq X$ as the fixed point set of a map homotopic to $f$ via a homotopy of pairs. Ng's results solved a problem raised by Schirmer in [11]. Since Ng's theory was never published, we include a sketch of his work for the convenience of the reader. We conclude by extending Ng's results to the case where $\Phi$ is a closed, locally contractible subset of $X$ (Theorem 5.3).

It is assumed that the reader is familiar with the general definitions and techniques of Nielsen theory, as in $[1,7]$.

\section{Neighborhood by-passing}

Let $X$ be a compact, connected polyhedron and $\Phi$ a subset of $X$. We say $\Phi$ can be by-passed in $X$ if every path in $X$ with endpoints in $X-\Phi$ is homotopic relative to the endpoints to a path in $X-\Phi$.

The notion of by-passing plays a key role in relative Nielsen theory and in realizing fixed point sets. Currently, we wish to extend Theorem 1.3 to the case where $\Phi$ is a locally contractible subset, but not necessarily a polyhedron (Theorem 3.5). To do so, we require a property that is closely related to by-passing. This property is the subject of the next definition.

Definition 2.1. A subset $\Phi$ of a topological space $X$ can be neighborhood by-passed if there exists an open set $V$ in $X$, containing $\Phi$, such that $V$ can be by-passed.

If $\Phi$ is chosen to be by-passed, the next theorem suggests that adding the requirement that $\Phi$ also be neighborhood by-passed does not affect our choice of $\Phi$.

Theorem 2.2. If $X$ is a compact, connected polyhedron, $\Phi \subseteq X$ is a closed, locally contractible subset, and if $\Phi$ can be by-passed, then $\Phi$ can be neighborhood by-passed.

Proof [3]. We prove this theorem in two steps. First we show that for any open neighborhood $U$ of $\Phi$, there exists a closed neighborhood $N \subset U$ of $\Phi$, with $X-N$ path connected. We then show that this neighborhood $N$ can be chosen to be by-passed in $X$.

Step 1. Given an open neighborhood $U$ of $\Phi$, there exists $N \subset U$, a closed neighborhood of $\Phi$, with $X-N$ path connected. Let $U \subset X$ be any open neighborhood of $\Phi$. Choose a closed neighborhood $M$ of $\Phi$, contained in $U$. Then $X-U$ can be covered by finitely many components of $X-M$. (This follows from compactness since $X-U$ is closed in $X$ and therefore compact.)

Since $\Phi$ can be by-passed in $X$, we can connect each pair of these components by a path in $X-\Phi$. In particular, for each pair of components $M_{i}^{\prime}$ and $M_{j}^{\prime}$ of $X-M$, choose points $x_{i} \in M_{i}^{\prime}$ and $x_{j} \in M_{j}^{\prime}$ and choose a path

$$
p_{i j}: I \longrightarrow(X-\Phi)
$$


4 Fixed point sets of maps homotopic to a given map

with

$$
p_{i j}(0)=x_{i}, \quad p_{i j}(1)=x_{j}
$$

( $x_{i}$ and $x_{j}$ can lie either in $U$ or its complement).

Next we find a closed neighborhood $K$ of $\Phi$, contained in $M$, such that $K$ misses all the paths $p_{i j}$. This is possible since

$$
(X-\operatorname{Int}(M)) \cup\left(\left\{p_{i j}\right\}\right)
$$

is compact (where $\operatorname{Int}(M)$ denotes the interior of $M$ ).

We will prove that there is exactly one path component of the complement of such $K$ which contains $X-U$.

First, observe that each component $M_{i}^{\prime}$ of $X-M$ must lie in a single component of $X-K$. If this was false, then for each component $K_{j}^{\prime}$ of $X-K$ which intersects $M_{i}^{\prime}$, we could write $M_{i}^{\prime}$ as a disjoint union of clopen sets,

$$
M_{i}^{\prime}=\bigsqcup_{j}\left(M_{i}^{\prime} \cap K_{j}^{\prime}\right)
$$

contrary to the connectedness of $M_{i}^{\prime}$.

Now suppose there exist two different components $M_{i}^{\prime}$ and $M_{j}^{\prime}$ of $X-M$, lying in different components of $X-K$. Then the path $p_{i j}$, as defined above, lies entirely within $X-K$ (by definition of $K$ ). But $p_{i j}$ must also intersect the two components of $X-K$, thus contradicting the connectedness of paths. Therefore, $M_{i}^{\prime}$ and $M_{j}^{\prime}$ (and hence all components of $X-M)$ lie in a single component of $X-K$. This component therefore contains $X-U$.

Finally, let $W$ be the path component of $X-K$ containing $X-U$. We have

$$
X-U \subset W \subset X-K,
$$

and hence

$$
\Phi \subset K \subset X-W \subset U
$$

Define $N=X-W$. Then $N \subset U$ is a closed neighborhood of $\Phi$ with path connected complement.

Step 2. We can choose the closed neighborhood $N$ from Step 1 to be a subset that can be by-passed in $X$ : since $X$ is a compact, connected polyhedron, it has a finitely generated fundamental group at any basepoint. Choose a basepoint $a \in(X-\Phi)$ and finitely many generators (loops)

$$
\rho_{1}, \ldots, \rho_{n}: I \longrightarrow X
$$

of $\pi_{1}(X, a)$. As $\Phi$ can be by-passed, these loops may be homotoped off $\Phi$. Thus without a loss of generality, we can rename these generators

$$
\rho_{1}, \ldots, \rho_{n}: I \longrightarrow(X-\Phi)
$$


Let

$$
P=\bigcup_{i=1}^{n} \operatorname{Im}\left(\rho_{i}\right)
$$

be a compact subset of $X-\Phi$, where $\operatorname{Im}\left(\rho_{i}\right)$ denotes the image of the path $\rho_{i}$. Let $U$ be an open neighborhood of $\Phi$ with $U \cap P=\varnothing$.

Then any loop $\alpha$ in $X$ with basepoint $a \in X-\Phi$ can be expressed as a word consisting of a finite number of loops in $X-U$. Thus, $\alpha$ is homotopic to a loop in $X-U$.

Now as in Step 1, choose $N$ in $U$ having path connected complement. Then by [9, Theorem 5.2], $N$ may be by-passed. Choosing $V=\operatorname{Int}(N)$ completes the proof.

\section{Realizing subsets of ANRs as fixed point sets}

Our present goal is to show that if the subset $\Phi$ in Theorem 1.3 is chosen to be locally contractible, but not necessarily polyhedral, the results of this theorem still hold. In particular, every closed subset of $\Phi$ that intersects every component of $\Phi$ can be realized as the fixed point set of a map homotopic to $f$. We will prove this by constructing a subpolyhedron of $X$ that contains such $\Phi$ and also satisfies the hypotheses of Theorem 1.3.

Lemma 3.1. If $\Phi$ is a closed subset of a compact, connected polyhedron $X$ and $X-\Phi$ is not a 2-manifold, then there exists a closed neighborhood $N$ of $\Phi$ such that $X-N$ is not a 2-manifold.

Proof. Since $X-\Phi$ is not a 2 -manifold, there exists an element $x \in X-\Phi$ with the property that no neighborhood of $x$ is homeomorphic to the 2-disk.

Let $d$ denote distance in $X$ and suppose $d(x, \Phi)=\delta>0$. Then the closed $\delta / 2$ neighborhood $N$ of $\Phi$ satisfies the property that $X-N$ is not a 2-manifold.

Definition 3.2. Let $Y$ be a metric space with distance $d$ and choose a real-valued constant $\varepsilon>0$. Given any topological space $X$, two maps $f, g: X \rightarrow Y$ are $\varepsilon$-near if $d(f(x), g(x))<\varepsilon$ for every $x \in X$. A homotopy $H: X \times I \rightarrow Y$ is called an $\varepsilon$-homotopy if for any $x \in X$, $\operatorname{diam}(H(x \times I))<\varepsilon$.

Here we assume the usual definition of diameter: given a subset $A \subseteq X$ and the distance $d$ on $X, \operatorname{diam}(A)=\sup \{d(x, y) \mid x, y \in A\}$. Thus,

$$
\operatorname{diam}(H(x \times I))=\sup \left\{d\left(H(x, t), H\left(x, t^{\prime}\right)\right) \mid t, t^{\prime} \in I\right\} .
$$

Theorem 3.3 [4, Proposition 3.4, page 121]. If $X$ is a metric ANR and $\Phi$ is a closed ANR subspace of $X$, then for every $\varepsilon>0$, there exists an $\varepsilon$-homotopy $h_{t}: X \rightarrow X$ satisfying

(1) $h_{0}=i d_{X}$,

(2) $h_{t}(x)=x$ for all $x \in \Phi, t \in I$,

(3) there exists an open neighborhood $U$ of $\Phi$ in $X$ such that $h_{1}(U)=\Phi$. 
6 Fixed point sets of maps homotopic to a given map

The map $h_{t}$ is called a strong deformation retraction of the space $U$ onto the subspace $\Phi$. We also say $U$ strong deformation retracts onto $\Phi$.

Lemma 3.4. Let $f: X \rightarrow X$ be a self-map of a compact, connected polyhedron and let $\Phi$ be a closed subset of $X$. Assume that there exists a subset $B$ of $X$ such that $\Phi \subseteq B$ and $B$ strong deformation retracts onto $\Phi$. If $f$ satisfies $(\mathrm{C} 1)$ and $(\mathrm{C} 2)$ for $\Phi$, then $f$ satisfies $(\mathrm{C} 1)$ and (C2) for $B$.

Proof. To verify (C1) for $B$, let $R: B \times I \rightarrow B$ denote the strong deformation retraction from $B$ onto $\Phi$, and denote $R(b, t)=r_{t}(b)$ for any $b \in B, t \in I$. So $r_{0}(b)=b, r_{1}(b) \in \Phi$, and $\left.r_{t}\right|_{\Phi}=i d_{\Phi}$. We will construct a homotopy $H_{B}: B \times I \rightarrow X$ from $f \mid B$ to the inclusion $i: B \hookrightarrow X$.

Let $H: B \times I \rightarrow X$ be the composition

$$
H(b, t)= \begin{cases}f \circ r_{2 t}(b) & 0 \leq t \leq 1 / 2, \\ H_{\Phi}\left(r_{1}(b), 2 t-1\right) & 1 / 2 \leq t \leq 1\end{cases}
$$

where $H_{\Phi}$ is the homotopy given by $(\mathrm{C} 1)$ on $\Phi$. Then $f$ is homotopic to $r_{1}$ via $H$.

Next we can construct a homotopy $H_{B}: B \times I \rightarrow X$ as follows:

$$
H_{B}(b, t)= \begin{cases}H(b, 2 t) & 0 \leq t \leq 1 / 2, \\ R(b, 2-2 t) & 1 / 2 \leq t \leq 1\end{cases}
$$

Observe that $\left.f\right|_{B}$ is homotopic to the identity via $H_{B}$. Thus, $H_{B}$ gives the desired homotopy satisfying $(\mathrm{C} 1)$ for $B$.

To prove (C2), choose any essential fixed point class $\mathbb{F}$ of $f: X \rightarrow X$. As $f$ satisfies (C2) for $\Phi$, there exists a path $\alpha: I \rightarrow X$ with $\alpha(0) \in \mathbb{F}$ and $\alpha(1) \in \Phi \subseteq B$, whence $\alpha(1) \in B$.

We show that the homotopy $H_{B}: B \times I \rightarrow X$ constructed above can be viewed as an extension of $H_{\Phi}: \Phi \times I \rightarrow X$. To see this, note that since $R: B \times I \rightarrow B$ is a strong deformation retraction, for any $x \in \Phi$,

$$
\begin{gathered}
H_{B}(x, t)= \begin{cases}H(x, 2 t) & 0 \leq t \leq 1 / 2, \\
x & 1 / 2 \leq t \leq 1,\end{cases} \\
H(x, t)= \begin{cases}f \circ r_{2 t}(x)=f(x) & 0 \leq t \leq 1 / 2, \\
H_{\Phi}(x, 2 t-1) & 1 / 2 \leq t \leq 1 .\end{cases}
\end{gathered}
$$

Thus for any $x \in \Phi$,

$$
H_{B}(x, t)= \begin{cases}f(x) & 0 \leq t \leq 1 / 4 \\ H_{\Phi}(x, 4 t-1) & 1 / 4 \leq t \leq 1 / 2 \\ x & 1 / 2 \leq t \leq 1\end{cases}
$$


and we say $\left.H_{B}\right|_{\Phi}$ is a reparametrization of $H_{\Phi}$. Then by defining a continuous map $\phi: I \rightarrow$ $I$ by

$$
\phi(s)= \begin{cases}0 & 0 \leq s \leq 1 / 4 \\ 4 s-1 & 1 / 4 \leq s \leq 1 / 2 \\ 1 & 1 / 2 \leq s \leq 1\end{cases}
$$

it is clear that $\left.H_{B}\right|_{\Phi}=H_{\Phi} \circ(i d \times \phi)$, where id denotes the identity map on $\Phi$ and

$$
(i d \times \phi)(x, s)=(x, \phi(s)),
$$

for any $x \in \Phi, s \in I$. Therefore, $H_{\Phi}$ is homotopic to $\left.H_{B}\right|_{\Phi}$ via the homotopy $\mathbb{T}:(X \times I) \times$ $I \rightarrow X$, defined by

$$
\mathbb{M}(x, t, s)=H_{\Phi}\left(x, \phi_{t}(s)\right),
$$

where

$$
\phi_{t}(s)=(1-t) \phi(s)+t s .
$$

Finally, since $f$ satisfies (C2) for $\Phi$, we know that for any essential fixed point class $\mathbb{F}$ of $f$, there exists a path $\alpha$ in $X$ with $\alpha(0) \in \mathbb{F}, \alpha(1) \in \Phi$, and

$$
\{\alpha(t)\} \backsim\{f \circ \alpha(t)\} *\left\{H_{\Phi}(\alpha(1), t)\right\} .
$$

From the above argument, $\left\{H_{\Phi}(\alpha(1), t)\right\} \backsim\left\{H_{B}(\alpha(1), t)\right\}$. Therefore,

$$
\{\alpha(t)\} \backsim\{f \circ \alpha(t)\} *\left\{H_{B}(\alpha(1), t)\right\}
$$

and $f$ satisfies (C2) for $B$.

As a consequence of the above results, we are now able to extend Theorem 1.3 to the case where $\Phi$ is locally contractible.

THeOREM 3.5. Let $f: X \rightarrow X$ be a self-map of a compact connected polyhedron without a local cutpoint. Let $\Phi$ be a closed, locally contractible subspace of $X$ satisfying

(1) $X-\Phi$ is not a 2-manifold,

(2) $f$ satisfies (C1) and (C2) for $\Phi$,

(3) $\Phi$ can be by-passed.

Then for every closed subset $\Gamma$ of $\Phi$ that has nonempty intersection with every component of $\Phi$, there exists a map $g$ homotopic to $f$ with Fix $g=\Gamma$. In particular, if $\Phi$ is connected, then every closed subset of $\Phi$ (including $\Phi$ itself) is the fixed point set of a map homotopic to $f$.

The proof of this theorem requires a polyhedral construction known as the star cover of a subset. Let $K$ be a triangulation of $X$. We write $X=|K|$. Then for any vertex $v$ of $K$, define the star of $v$, denoted $\operatorname{St}_{K}(v)$, to be the union of all closed simplices of which $v$ is a vertex. Then for any subspace $\Phi \subseteq X$, the star cover of $\Phi$ is

$$
\mathrm{St}_{K}(\Phi)=\bigcup_{v \in \Phi} \mathrm{St}_{K}(v)
$$




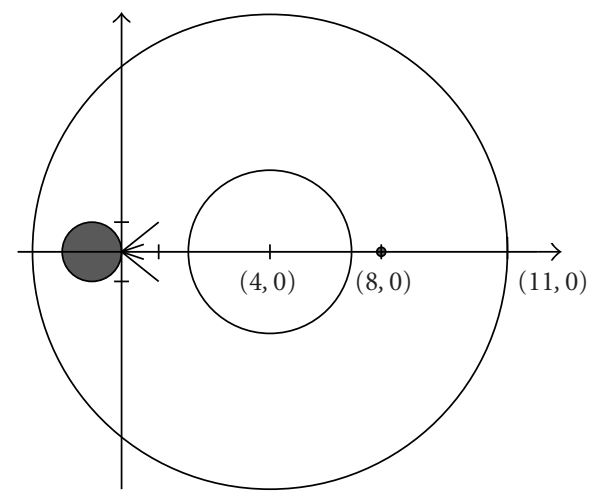

Figure 3.1. A locally contractible fixed point set.

Proof of Theorem 3.5. We can assume $\Phi \neq \varnothing$ as, otherwise, this theorem reduces to [10, Lemma 3.1]. Since $X$ is a polyhedron, let $K$ be a triangulation of $X=|K|$. By [2, Proposition 8.12, page 83], $\Phi$ is a finite-dimensional ANR. Thus, Theorem 3.3 gives an open neighborhood $U$ of $\Phi$ that strong deformation retracts onto $\Phi$. Since $\Phi$ can be by-passed, Theorem 2.2 implies that there exists another open neighborhood $V$ of $\Phi$ such that $V$ can be by-passed. The set $V$ may be chosen to lie inside $U$. Choose a star cover $\operatorname{St}_{K^{\prime}}(\Phi)$ of $\Phi$ with respect to a sufficiently small subdivision $K^{\prime}$ of $K$ such that $\mathrm{St}_{K^{\prime}}(\Phi) \subset V$. Then $(\mathrm{C} 1)$ and (C2) hold for $\mathrm{St}_{K^{\prime}}(\Phi)$ (Lemma 3.4). Further, the subdivision $K^{\prime}$ can be chosen small enough so that $X-\mathrm{St}_{K^{\prime}}(\Phi)$ is not a 2-manifold (Lemma 3.1).

By the construction of star covers, each component of $\mathrm{St}_{K^{\prime}}(\Phi)$ contains a component of $\Phi$. If every component of $\Phi$, in turn, intersects a given closed subset $\Gamma \subset \Phi$, then each component of the star cover intersects $\Gamma$. As star covers are themselves polyhedra, the result follows from Theorem 1.3.

We close this section with an example of a self-map $f$ on a compact, connected polyhedron $X$, with a locally contractible subset $\Phi$ that is not a finite polyhedron, for which there exists $g$ homotopic to $f$ with Fix $g=\Phi$.

Example 3.6. Consider the space

$$
X=\left\{(x, y) \in \mathbb{R}^{2} \mid 4 \leq(x-4)^{2}+y^{2} \leq 49\right\}
$$

the annulus in $\mathbb{R}^{2}$ centered at the point $(4,0)$, with outer radius 7 and inner radius 2 (see Figure 3.1). Let $f: X \rightarrow X$ be the map flipping $X$ over the $x$-axis. That is, $f(x, y)=(x,-y)$.

Clearly $\operatorname{Fix}(f)$ lies on the $x$-axis and $f$ has exactly two fixed point classes,

$$
\mathbb{F}_{1}=\{(x, 0) \mid-3 \leq x \leq 2\}, \quad \mathbb{F}_{2}=\{(x, 0) \mid 6 \leq x \leq 11\}
$$


We define $\Phi=D \cup Z \cup\{(8,0)\}$ where

$$
\begin{gathered}
D=\left\{(x, y) \mid(x+1)^{2}+y^{2} \leq 1\right\}, \\
Z=\bigcup_{k=1}^{\infty}\left(\left[0, z_{k}\right] \cup\left[0, z_{-k}\right]\right) .
\end{gathered}
$$

For each positive integer $k,\left[0, z_{k}\right]$ denotes the line segment in $\mathbb{R}^{2}$ from the point $(0,0)$ to the point $\left(1 / k, 1 / k^{2}\right)$, and $\left[0, z_{-k}\right]$ is the line segment from $(0,0)$ to $\left(1 / k,-1 / k^{2}\right)$.

First we show that $\Phi$ is locally contractible. At the origin, a sufficiently small neighborhood contracts via straight lines. Also for each $k$, given any point on the line segment $\left[0, z_{k}\right]$, we can find a neighborhood that does not contain any other segment of $\Phi$, and hence contracts along the segment $\left[0, z_{k}\right]$. Lastly, it is clear that $D$ is itself locally contractible.

The subset $\Phi$ is also clearly closed and can be by-passed in $X$. Thus, it remains to be shown that $f$ satisfies $(\mathrm{C} 1)$ and $(\mathrm{C} 2)$ for $\Phi$.

To verify (C1), observe that $\Phi$ is homotopy equivalent to $\mathbb{F}_{1} \cup \mathbb{F}_{2}$. Let $r: \Phi \rightarrow \mathbb{F}_{1} \cup$ $\mathbb{F}_{2}$ and $s: \mathbb{F}_{1} \cup \mathbb{F}_{2} \rightarrow \Phi$, where $s \circ r \simeq i d_{\Phi}$ and $r \circ s \simeq i d_{\mathbb{F}_{1} \cup \mathbb{F}_{2}}$. We have the sequence of homotopies

$$
\left.f\right|_{\Phi}=\left.\left.f\right|_{\Phi} \circ i d_{\Phi} \simeq f\right|_{\Phi} \circ(s \circ r)=s \circ r \simeq i d_{\Phi},
$$

where the second equality holds true because $f$ is the identity map on $\mathbb{F}_{1} \cup \mathbb{F}_{2}$.

To prove (C2), we must find an appropriate path $\alpha_{i}$ for each class $\mathbb{F}_{i}(i=1,2)$. For $\mathbb{F}_{1}$, we can choose $\alpha_{1}$ to be the constant path at the point $(-1,0)$, and for $\mathbb{F}_{2}$ we can choose $\alpha_{2}$ to be the constant path at the point $(8,0)$. The point at which we define $\alpha_{i}$ is unimportant, provided that the point lies in the intersection of $\Phi$ with the fixed point class. It is clear that $\alpha_{i}(0) \in \mathbb{F}_{i}$ and $\alpha_{i}(1) \in \Phi$ for $i=1,2$. Moreover, the required homotopy holds trivially, thus proving $(\mathrm{C} 2)$.

Therefore by Theorem 3.5, $\Phi$ is the fixed point set of a map homotopic to $f$. It is clear that $\Phi$ is not a finite polyhedron, thus showing that there exist interesting sets that satisfy the hypotheses of Theorem 3.5, but do not satisfy the hypotheses of Theorem 1.3.

\section{Polyhedral fixed point sets of maps of pairs}

Given a compact polyhedral pair $(X, A)$, let $Z=\operatorname{cl}(X-A)$ denote the closure of $X-A$. For any subset $\Phi \subseteq X$, let $\Phi_{A}=A \cap \Phi$. We call $\left(\Phi, \Phi_{A}\right)$ a subset pair of $(X, A)$. For any map $f:(X, A) \rightarrow(X, A)$, denote the restriction $\left.f\right|_{A}$ as $f_{A}: A \rightarrow A$. We write $f \sim_{A} g$ if there exists a homotopy of pairs $H:(X, A) \times I \rightarrow(X, A)$ from $f$ to $g$ where $(X, A) \times I$ denotes the pair $(X \times I, A \times I)$. If $f \sim_{A} g$, it follows that $f_{A} \backsim g_{A}$ via the restriction of the homotopy to $A$.

In [8], Ng developed the following definition and theorems. As all the proofs can be found in [8], we provide only a sketch of each proof here. All references to (C1) and (C2) are to Schirmer's conditions, as stated in Definition 1.1.

Definition 4.1. Let $f:(X, A) \rightarrow(X, A)$ be a map of a compact polyhedral pair. The map $f$ satisfies conditions $\left(\mathrm{C1}^{\prime}\right)$ and $\left(\mathrm{C}^{\prime}\right)$ for a subset $\Phi \subseteq X$ if the following are satisfied 
(the symbol $\backsim$ denotes the usual homotopy of paths with endpoints fixed and $*$ the path product):

$\left(\mathrm{Cl}^{\prime}\right)$ there exists a homotopy $\tilde{H}:\left(\Phi, \Phi_{A}\right) \times I \rightarrow(X, A)$ from $\left.f\right|_{\Phi}$ to the inclustion $i$ : $\Phi \hookrightarrow X$ and the map $f_{A}$ satisfies (C1) and (C2) for $\Phi_{A}$ in $A$ where $H_{\Phi_{A}}=\left.\tilde{H}\right|_{\Phi_{A} \times I}$, $\left(\mathrm{C} 2^{\prime}\right)$ for every essential fixed point class $\mathbb{F}$ of $f$ intersecting $Z$, there exists a path $\alpha: I \rightarrow$ $Z$ with $\alpha(0) \in \mathbb{F} \cap Z, \alpha(1) \in \Phi$, and

$$
\{\alpha(t)\} \backsim\{f \circ \alpha(t)\} *\{\tilde{H}(\alpha(1), t)\} .
$$

Theorem 4.2. Let $f:(X, A) \rightarrow(X, A)$ be a map of a compact polyhedral pair. If $f$ satisfies conditions $\left(\mathrm{C}^{\prime}\right)$ and $\left(\mathrm{C} 2^{\prime}\right)$ for a subset $\Phi \subseteq X$, then $f$ satisfies $(\mathrm{C} 1)$ and $(\mathrm{C} 2)$ for $\Phi$.

Sketch of proof. First observe that by choosing $A$ to be the empty set, $\left(\mathrm{Cl}^{\prime}\right)$ implies $(\mathrm{C} 1)$. To prove (C2), choose any essential fixed point class $\mathbb{E}$ of $f$. We can write

$$
\mathbb{F}=\mathbb{F}_{A} \cup \mathbb{F}_{Z},
$$

where

$$
\mathbb{F}_{A}=\mathbb{F} \cap A, \quad \mathbb{F}_{Z}=\mathbb{F}-\operatorname{Int}(A)=\mathbb{F} \cap Z .
$$

By $\left[5\right.$, Theorem 1.1], there exists an integer-valued index $\operatorname{ind}_{A}\left(f, \mathbb{F}_{Z}\right)$ such that

$$
\operatorname{ind}_{A}\left(f, \mathbb{F}_{Z}\right)=\operatorname{ind}(f, \mathbb{F})-\operatorname{ind}\left(f_{A}, \mathbb{F}_{A}\right) \text {, }
$$

where "ind" denotes the classical fixed point index.

Suppose $\operatorname{ind}_{A}\left(f, \mathbb{F}_{Z}\right) \neq 0$. Write

$$
\mathbb{F}_{Z}=\mathbb{F}_{1} \cup \cdots \cup \mathbb{F}_{k}
$$

where for each $i$ between 1 and $k, \mathbb{F}_{i}$ denotes the intersection of $\mathbb{F}$ with a path component of $Z$. It follows from [5] that $\operatorname{ind}_{A}\left(f, \mathbb{F}_{Z}\right)=\sum_{i=1}^{k} \operatorname{ind}_{A}\left(f, \mathbb{F}_{i}\right)$. Then since $\operatorname{ind}_{A}\left(f, \mathbb{F}_{Z}\right) \neq 0$, there exists at least one $i$ for which $\operatorname{ind}_{A}\left(f, \mathbb{F}_{i}\right) \neq 0$. This $\mathbb{F}_{i}$ can be written as a finite union of fixed point classes of $f$ intersecting $Z$. At least one of these classes must be an essential class of $f$ intersecting $Z$. Denote this class as $\mathbb{C}$. Then by $\left(\mathrm{C}^{\prime}\right)$, there exists a path $\alpha: I \rightarrow Z$ with $\alpha(0) \in \mathbb{G} \subseteq \mathbb{F}, \alpha(1) \in \Phi$ and

$$
\{\alpha(t)\} \backsim\{f \circ \alpha(t)\} *\{\tilde{H}(\alpha(1), t)\},
$$

thus proving $(\mathrm{C} 2)$ for this case.

Next suppose that $\operatorname{ind}_{A}\left(f, \mathbb{F}_{Z}\right)=0$. Then $\operatorname{ind}\left(f_{A}, \mathbb{F}_{A}\right) \neq 0$, implying that $\mathbb{F}_{A}$ is an essential fixed point class of $f_{A}$. From $\left(\mathrm{Cl}^{\prime}\right)$ there exists a path $\alpha: I \rightarrow A$ with $\alpha(0) \in \mathbb{F}_{A}$, $\alpha(1) \in \Phi_{A} \subset \Phi$, and

$$
\begin{aligned}
\{\alpha(t)\} & \backsim\left\{f_{A} \circ \alpha(t)\right\} *\left\{H_{\Phi}(\alpha(1), t)\right\} \\
& =\{f \circ \alpha\} *\{\tilde{H}(\alpha(1), t)\},
\end{aligned}
$$

which proves (C2). 
Corollary 4.3. Let $f:(X, A) \rightarrow(X, A)$ be a map of a compact polyhedral pair. If $f$ satisfies conditions $\left(\mathrm{C}^{\prime}\right)$ and $\left(\mathrm{C} 2^{\prime}\right)$ for a subset $\Phi \subseteq X$, then $\Phi$ has at least $N(f)$ components and $\Phi_{A}$ has at least $N\left(f_{A}\right)$ components.

It is worthwhile to observe that in some cases, $\left(\mathrm{C}^{\prime}\right)$ is easy to check. In particular, $f$ satisfies $\left(C 2^{\prime}\right)$ for $\Phi \subseteq X$ if any of the following is satisfied ([8]):

(1) $N\left(\left.f\right|_{Z}\right)=0$,

(2) $X$ is simply connected,

(3) $\Phi \subseteq$ Fix $f \cap Z$ and $\Phi$ intersects every essential fixed point class of $f$ on $Z$.

Theorem 4.4 (necessity). Let $f:(X, A) \rightarrow(X, A)$ be a map of a compact polyhedral pair and let $\Phi$ be a subspace of $X$. If there exists a map $g \sim_{A} f$ with Fix $g=\Phi$, then $f$ satisfies $\left(\mathrm{C}^{\prime}\right)$ and $\left(\mathrm{C} 2^{\prime}\right)$ for $\Phi$.

Sketch of proof. Let $H$ denote the homotopy of pairs from $f$ to $g$. It is clear that by letting $\tilde{H}=\left.H\right|_{\Phi \times I}$ and applying [10, Theorem 2.1], $f$ satisfies $\left(\mathrm{Cl}^{\prime}\right)$.

To prove $\left(\mathrm{C}^{\prime}\right)$, choose any essential fixed point class $\mathbb{F}$ of $f$ intersecting $Z$. It follows from [13, Theorem 2.7] that there exists an essential fixed point class $\mathbb{C}$ of $g$ intersecting $Z$, to which $\mathbb{F}$ is $H$-related. Thus, there exists a path $\alpha: I \rightarrow Z$ with $\alpha(0) \in \mathbb{F}, \alpha(1) \in \Phi$, and

$$
\begin{aligned}
\{\alpha(t)\} & \backsim\{H(\alpha(t), t)\} \\
& \backsim\{H(\alpha(t), 0)\} *\{H(\alpha(1), t)\} \\
& =\{f \circ \alpha(t)\} *\{\tilde{H}(\alpha(1), t)\} .
\end{aligned}
$$

Therefore $f$ satisfies $\left(\mathrm{C}^{\prime}\right)$ for $\Phi$.

Theorem 4.5 (Ng's finiteness theorem). Let $f:(X, A) \rightarrow(X, A)$ be a map of a compact polyhedral pair in which $X$ and $A$ have no local cutpoints. Suppose $\left(\Phi, \Phi_{A}\right)$ is a subpolyhedral pair such that

(1) $A-\Phi_{A}$ is not a 2-manifold,

(2) $\Phi_{A}$ can be by-passed in $A$,

(3) $f$ satisfies $\left(\mathrm{Cl}^{\prime}\right)$ for $\Phi$.

Then there exists a map $g \sim_{A} f$ via a homotopy $H:(X, A) \times I \rightarrow(X, A)$ that extends $\tilde{H}$ such that Fix $g=\Phi \cup Z_{o}$, where $Z_{o}$ is a finite subset of $X-A$ and each point of $Z_{o}$ lies in the interior of a maximal simplex of $X$.

Sketch of proof. To construct the homotopy $H$, we will build three homotopies $H_{1}, H_{2}$, and $H_{3}$, and take their composition.

From conditions (1)-(3), we can apply [10, Lemma 3.1] to show that there exists a map $g_{1, A}$ homotopic to $f_{A}$ with Fix $g_{1, A}=\Phi_{A}$ via a homotopy $H_{A}: A \times I \rightarrow A$ that is an extension of $\left.\tilde{H}\right|_{\Phi_{A} \times I}$. Consider the homotopy $H_{1, A}:(A \cup \Phi, A) \times I \rightarrow(X, A)$ defined by

$$
H_{1, A}(x, t)= \begin{cases}H_{A}(x, t) & (x, t) \in A \times I, \\ \tilde{H}(x, t) & (x, t) \in \Phi \times I .\end{cases}
$$


By the homotopy extension property, there is a homotopy $H_{1}:(X, A) \times I \rightarrow(X, A)$ that is an extension of $H_{1, A}$; let $f_{1}(x)=H_{1}(x, 1)$. It is easy to check that $\Phi \subseteq \operatorname{Fix} f_{1}$, Fix $\left.f_{1}\right|_{A}=\Phi_{A}$, and $H_{1}$ extends $\tilde{H}$.

To construct $H_{2}$, choose a strong deformation retraction $R: \operatorname{St}(A \cup \Phi) \times I \rightarrow \operatorname{St}(A \cup$ $\Phi)$ of a star cover of $A \cup \Phi$ onto the set $A \cup \Phi$. We will abbreviate $\mathrm{St}_{A \cup \Phi}$ for the star cover $\operatorname{St}(A \cup \Phi)$. We can define $H_{2}:(X, A) \times I \rightarrow(X, A)$ to be an extension of the composition $f_{1} \circ R:\left(\operatorname{St}_{A \cup \Phi}, A\right) \times I \rightarrow(X, A)$. Setting $f_{2}(x)=H_{2}(x, t)$, it is easy to check that Fix $\left.f_{2}\right|_{A}=$ $\Phi_{A}$ and Fix $f_{2}=A \cup\left(X-\mathrm{St}_{A \cup \Phi}\right)$.

By a careful application of the Hopf construction, we can find a map $f_{3}: \operatorname{cl}\left(X-\mathrm{St}_{A \cup \Phi}\right)$ $\rightarrow X$ that is $\varepsilon$-homotopic to $\left.f_{2}\right|_{\mathrm{cl}\left(X-S t_{A \cup \Phi}\right)}$, where $f_{3}$ has only a finite number of fixed points, each lying in the interior of a maximal simplex of $X$. Let $H_{3, \mathrm{cl}}: \operatorname{cl}\left(X-\mathrm{St}_{A \cup \Phi}\right) \times I \rightarrow X$ denote the homotopy from $\left.f_{2}\right|_{\mathrm{cl}\left(X-\mathrm{St}_{A \cup \Phi}\right)}$ to $f_{3}$. We construct another homotopy $H_{3}^{\prime}$ : $\left(\partial\left(\mathrm{St}_{A \cup \Phi}\right) \cup A \cup \Phi, A\right) \times I \rightarrow(X, A)$ as follows:

$$
H_{3}^{\prime}(x, t)= \begin{cases}H_{3, \mathrm{cl}}(x, t) & (x, t) \in \partial\left(\mathrm{St}_{A \cup \Phi}\right), \\ f_{2}(x) & (x, t) \in A \cup \Phi .\end{cases}
$$

Then $H_{3}^{\prime}$ can be extended to a homotopy $H_{3, \mathrm{St}}:\left(\mathrm{St}_{A \cup \Phi}, A\right) \times I \rightarrow(X, A)$. Finally, we define $H_{3}:(X, A) \times I \rightarrow(X, A)$ by

$$
H_{3}(x, t)= \begin{cases}H_{3, \mathrm{St}}(x, t) & x \in \mathrm{St}_{A \cup \Phi}, \\ H_{3, \mathrm{cl}}(x, t) & x \in \mathrm{cl}\left(X-\mathrm{St}_{A \cup \Phi}\right) .\end{cases}
$$

One can check that if we let $H$ be the composition of the homotopies $H_{1}, H_{2}$, and $H_{3}$ and define $g(x)=H(x, 1)$, we complete the proof.

Theorem 4.6 (Ng's sufficiency theorem \#1). Let $f:(X, A) \rightarrow(X, A)$ be a map of a compact polyhedral pair in which $X$ and $A$ have no local cutpoints. Suppose $\left(\Phi, \Phi_{A}\right)$ is a subpolyhedral pair such that

(1) $A-\Phi_{A}$ and all components of $X-(A \cup \Phi)$ are not 2-manifolds,

(2) $f$ satisfies $\left(\mathrm{C1}^{\prime}\right)$ and $\left(\mathrm{C} 2^{\prime}\right)$ for $\Phi$,

(3) $\Phi_{A}$ can be by-passed in $A$, $\Phi$ can be by-passed in $X-A$ and $\partial A$ can be by-passed in $Z$. Then there exists a map $g \sim_{A} f$ with Fix $g=\Phi$.

Sketch of proof. From Theorem 4.5, there exists a map $g_{1} \sim_{A} f$ via a homotopy $H:(X$, $A) \times I \rightarrow(X, A)$ that extends $\tilde{H}$ with Fix $g_{1}=\Phi \cup Z_{o}$, where $Z_{o}$ is a finite subset of $X-A$ and each point of $Z_{o}$ lies in the interior of a maximal simplex of $X$. To construct the desired map $g$, we use a sequence of homotopies relative to $A \cup \Phi$.

Using techniques from $[6,9]$, one can show that the following procedures are possible in this scenario.

(1) Given any two points $x, y \in$ Fix $g_{1} \cap(X-A)$ that lie in the same fixed point class of $g_{1}$ intersecting $Z$, we can delete the point $x$ from Fix $g_{1}$ by an appropriate homotopy. This requires the assumption that every component of $X-(A \cup \Phi)$ is not a 2-manifold.

(2) If $x \in$ Fix $g_{1} \cap(X-A)$ and $y$ is any point in $Z \cap \Phi$ that lies in the same fixed point class as $x$, we can delete the point $x$ from Fix $g_{1}$ by an appropriate homotopy. In 
addition to the first assumption, this requires that $\partial A$ can be by-passed in $Z$ and $\Phi$ can be by-passed in $X-A$.

(3) Any point $x \in \operatorname{Fix}_{1} \cap(X-A)$ with ind $(f, x)=0$ can be removed in the usual way.

After a finite number of applications of the above procedures, we achieve a new map $g \sim_{A} f$. If $g$ is fixed point free on $X-(\Phi \cup A)$, we are done. If Fix $g \cap(X-(\Phi \cup A)) \neq$ $\varnothing$, then any point $x \in$ Fix $g \cap(X-(\Phi \cup A))$ forms an entire essential fixed point class of $g$. A slight modification of the proof of [10, Lemma 3.1] shows that this scenario is impossible.

In the original statement of Theorem 4.6, $\mathrm{Ng}$ required that no component of $A-\Phi_{A}$ be a 2-manifold. However, this assumption is not required for the proof and therefore omitted.

Theorem 4.7 (Ng's sufficiency theorem \#2). Let $f:(X, A) \rightarrow(X, A)$ be a map of a compact polyhedral pair in which $X$ and $A$ have no local cutpoints. Suppose $\left(\Phi, \Phi_{A}\right)$ is a subpolyhedral pair such that

(1) $A-\Phi_{A}$ and all components of $X-(A \cup \Phi)$ are not 2-manifolds,

(2) $f$ satisfies $\left(\mathrm{C1}^{\prime}\right)$ and $\left(\mathrm{C} 2^{\prime}\right)$ for $\Phi$,

(3) $\Phi_{A}$ can be by-passed in $A$, $\Phi$ can be by-passed in $X-A$, and $\partial A$ can be by-passed in $Z$. Then for every closed subset $\Gamma$ of $\Phi$ that has nonempty intersection with every component of $\Phi_{A}$ and every component of $\Phi \cap Z$, there exists a map $g \sim_{A} f$ with Fix $g=\Gamma$.

Sketch of proof. Let $K$ be a triangulation of $X=|K|$. As in the proof of [10, Theorem 3.2], we can find a subpolyhedron $N$ in a subdivision $K^{\prime}$ of $K$ such that $\left.f\right|_{N}$ is a proximity map with only a finite number of fixed points, all lying in $\Gamma \subseteq \operatorname{Int}(N)$. Let $\alpha(x, y, t)$ be defined as in [1, Lemma 1, page 124]. Define a homotopy $H_{N}:(N, N \cap A) \times I \rightarrow(X, A)$ by

$$
H_{N}(x, t)=\alpha(x, f(x), 1-t(1-d(x, \Gamma)))
$$

where $d$ denotes the usual distance function. It is not difficult to check that $H_{N}$ is a special homotopy (cf. [6, page 751]) on $\partial N \times I$ and that Fix $H_{N}(x, 1)=\Gamma$. Next, we can extend $H_{N}$ to a new homotopy $H:(X, A) \times I \rightarrow(X, A)$ that is special on $\mathrm{cl}(X-N)$. If we let $g(x)=H(x, 1)$, then $g \sim_{A} f$ and Fix $g=\Gamma$.

\section{Locally contractible fixed point sets of maps of pairs}

We wish to extend Ng's work (in particular, Theorem 4.7 ) to the case where $\Phi$ is locally contractible, but not necessarily a polyhedron. To do so, we first prove a useful lemma and theorem.

Lemma 5.1. Let $f:(X, A) \rightarrow(X, A)$ be a map of a compact polyhedral pair and let $\left(\Phi, \Phi_{A}\right)$ be a subset pair in which $\Phi$ is closed in $X$. Assume that there exists a subset $B$ of $X$ such that $\Phi \subseteq B$ and the pair $(B, B \cap A)$ strong deformation retracts onto $\left(\Phi, \Phi_{A}\right)$ via a retraction $R:(B, B \cap A) \times I \rightarrow(B, B \cap A)$. If $f$ satisfies $\left(\mathrm{C}^{\prime}\right)$ and $\left(\mathrm{C}^{\prime}\right)$ for $\Phi$, then $f$ satisfies $\left(\mathrm{C}^{\prime}\right)$ and $\left(\mathrm{C} 2^{\prime}\right)$ for $B$. 
14 Fixed point sets of maps homotopic to a given map

Proof. First observe that since $f$ satisfies $\left(\mathrm{C1}^{\prime}\right)$ for $\Phi, f_{A}$ satisfies $(\mathrm{C} 1)$ and $(\mathrm{C} 2)$ for $\Phi_{A}$ in $A$. Then Lemma 3.4 shows that $f_{A}$ satisfies $(\mathrm{C} 1)$ and (C2) for $B \cap A$. This proves the second statement in $\left(\mathrm{Cl}^{\prime}\right)$. A simple modification of the proof of Lemma 3.4 proves the first statement in $\left(\mathrm{C}^{\prime}\right)$ and also proves that $f$ satisfies $\left(\mathrm{C}^{\prime}\right)$ for $B$.

Theorem 5.2. Let $f:(X, A) \rightarrow(X, A)$ be a map of a compact polyhedral pair. Suppose $\left(\Phi, \Phi_{A}\right)$ is a subset pair in which both $\Phi$ and $\Phi_{A}$ are closed, locally contractible subsets of $X$. Then there exists a subset $B$ of $X$ such that $\Phi \subseteq B$ and the pair $(B, B \cap A)$ strong deformation retracts onto $\left(\Phi, \Phi_{A}\right)$ via a retraction of pairs $\mathscr{R}_{t}:(B, B \cap A) \rightarrow(B, B \cap A)$.

Proof. We will construct three homotopies and then take their composition to obtain an explicit strong deformation retraction of pairs. First, we must establish some terminology.

Since $X$ itself is an ANR embedded in Euclidean space, there exists a neighborhood $V \subset \mathbb{R}^{n}(n>0)$ of $X$ that strong deformation retracts onto $X$. Let

$$
\rho_{t}: V \rightarrow X
$$

denote this strong deformation retraction. The subset $\Phi$ is also a finite-dimensional ANR ([2, Proposition 8.12, page 83]). Thus, there exists a neighborhood $U \subset X$ that strong deformation retracts onto $\Phi$. Let

$$
\varphi_{t}: U \longrightarrow \Phi
$$

denote this strong deformation retraction. Define

$$
\varepsilon=\min \left(d\left(X, V^{c}\right), d\left(\Phi, U^{c}\right)\right)
$$

where $V^{c}$ and $U^{c}$ denote the complements of $V$ and $U$ in $\mathbb{R}^{n}$ and $X$, respectively.

Choose any three positive real numbers $\varepsilon_{1}, \varepsilon_{2}$, and $\varepsilon_{3}$ so that

$$
\varepsilon_{1}+\varepsilon_{2}+\varepsilon_{3}<\varepsilon
$$

The subsets $\Phi$ and $\Phi_{A}$ are both finite-dimensional ANR's. Thus, there exist neighborhoods $U_{1}, U_{2} \subset X$ and strong deformation retractions $R_{t}: U_{1} \rightarrow \Phi$ and $r_{t}: U_{2} \rightarrow \Phi$ that are $\varepsilon_{1}$ - and $\varepsilon_{2}$-homotopies, respectively (Theorem 3.3). In other words, for each $x \in U_{1}$,

$$
d\left(R_{t}(x), R_{t^{\prime}}(x)\right)<\varepsilon_{1}, \quad \text { for any } t, t^{\prime} \in I
$$

and for each $x \in U_{2}$,

$$
d\left(r_{t}(x), r_{t^{\prime}}(x)\right)<\varepsilon_{2}, \quad \text { for any } t, t^{\prime} \in I
$$

Notice that although both $R_{t}$ and $\varphi_{t}$ are strong deformation retractions of neighborhoods of $\Phi$ onto itself, we neither require that $U_{1}=U$ nor that $\left.R_{t}\right|_{U_{1} \cap U}=\left.\varphi_{t}\right|_{U_{1} \cap U}$.

Next define $\delta_{1}, \delta_{2}>0$ by

$$
\delta_{1}=d\left(\Phi, X-U_{1}\right), \quad \delta_{2}=d\left(\Phi_{A}, X-U_{2}\right),
$$


and let $\Delta=\min \left(\delta_{1}, \delta_{2}\right)$. Define $B$ to be a $\Delta$-neighborhood of $\Phi$. Then $\Phi \subset B \subseteq U_{1}$ and $\Phi_{A} \subset B \cap A \subseteq U_{2}$. By restricting $R_{t}$ to $B$ and $r_{t}$ to $B \cap A$, we can view these maps as strong deformation retractions of $B$ onto $\Phi$ and of $B \cap A$ onto $\Phi_{A}$, respectively.

Next, since the subset $A$ is a subpolyhedron of $X$, it must also be a finite-dimensional ANR. Thus, there exists a neighborhood $W \subset X$ containing $A$ and a strong deformation retraction $\psi_{t}: W \rightarrow A$ that is an $\varepsilon_{3}$-homotopy. For ease of notation, we will let $x^{\prime}=\psi_{1}(x)$ for any $x \in W$.

Let us choose $\Omega=d(\operatorname{cl}(B \cap A), X-W)$ and define a set

$$
C=\{x \in B-B \cap A \mid d(x, \operatorname{cl}(B \cap A)) \leq \Omega\} .
$$

For any $x \in C$, let

$$
d(x, \operatorname{cl}(B \cap A))=s \leq \Omega, \quad d(x, \Phi)=q<\Delta .
$$

Finally, we let $\beta=\max (\Omega, \Delta)>0$, and we define our first homotopy to be the map $H_{t}$ : $(B, B \cap A) \rightarrow(B, B \cap A)$ defined by

$$
H_{t}(x)= \begin{cases}x & x \in B-C, \\ \rho_{1-t}\left(\left\langle\psi_{t}(x), x\right\rangle \frac{1}{\beta}\right) & x \in C, q=s, \\ \rho_{1-t}\left(\left[\left\langle\psi_{t}(x), x\right\rangle \frac{q}{\beta}+(s-q) x\right] \frac{1}{s}\right) & x \in C, q<s, \\ \rho_{1-t}\left(\left[\left\langle\psi_{t}(x), x\right\rangle \frac{s}{\beta}+(q-s) \psi_{t}(x)\right] \frac{1}{q}\right) & x \in C, q>s,\end{cases}
$$

where $\left\langle\psi_{t}(x), x\right\rangle=(\beta-s) \psi_{t}(x)+s x$.

We must check that $H_{t}$ is defined on $C$. By the definition of $C$, if $x \in C$ then $x \in$ $B \cap W$. Thus, $\psi_{t}(x)$ is defined. Next, since $s \leq \Omega \leq \beta$, for each $t \in I$ the expression $\left\langle\psi_{t}(x)\right.$, $x\rangle(1 / \beta)=\left[(\beta-s) \psi_{t}(x)+s x\right](1 / \beta)$ represents a point lying on the straight path in $\mathbb{R}^{n}$ between $\psi_{t}(x)$ and $x$. Since $\psi_{t}(x)$ is an $\varepsilon_{3}$-homotopy, the length of this path must be less than $\varepsilon_{3}$. Moreover,

$$
\varepsilon_{3}<\varepsilon=\min \left(d\left(X, V^{c}\right), d\left(\Phi, U^{c}\right)\right)
$$

and $\psi_{t}(x), x \in X$. Thus all points on this straight path must lie in $V$, whence $\rho_{1-t}\left(\left\langle\psi_{t}(x)\right.\right.$, $x>(1 / \beta)$ exists.

To see that $H_{t}(x)$ is defined for $x \in C$ with $q<s$, observe that for each $t \in I$ the expression

$$
\left[\left\langle\psi_{t}(x), x\right\rangle \frac{q}{\beta}+(s-q) x\right] \frac{1}{s}=\left[q\left[(\beta-s) \psi_{t}(x)+s x\right] \frac{1}{\beta}+(s-q) x\right] \frac{1}{s}
$$

represents a point lying on the straight path in $\mathbb{R}^{n}$ between $x$ and some point lying between $\psi_{t}(x)$ and $x$. Thus, the length of the path in (5.12) must be less than or equal to the length of the straight path between $\psi_{t}(x)$ and $x$. In short, all points in this expression 
must lie in $V$. Thus, $\rho_{1-t}\left(\left[\left\langle\psi_{t}(x), x\right\rangle(q / \beta)+(s-q) x\right](1 / s)\right)$ is defined. A similar argument holds for $x \in C$ with $q>s$. Therefore, $H_{t}(x)$ is defined for all $x \in C$.

It is straightforward to check that $H_{t}$ is continuous, $H_{0}=i d_{B}$, and $H_{t}$ is a homotopy of pairs.

We define the second homotopy of pairs in the composition to be $J_{t}:(B, B \cap A) \rightarrow$ $(\Phi, \Phi \cap A)$ such that

$$
J_{t}(x)= \begin{cases}r_{t}(x) & x \in B \cap A, \\ R_{t}(x) & x \in(B-B \cap A)-C, \\ \rho_{t}\left(\left\langle r_{t}\left(x^{\prime}\right), R_{t}(x)\right\rangle \frac{1}{\beta}\right) & x \in C, q=s, \\ \rho_{t}\left(\left[\left\langle r_{t}\left(x^{\prime}\right), R_{t}(x)\right\rangle \frac{q}{\beta}+(s-q) R_{t}(x)\right] \frac{1}{s}\right) & x \in C, q<s, \\ \rho_{t}\left(\left[\left\langle r_{t}\left(x^{\prime}\right), R_{t}(x)\right\rangle \frac{s}{\beta}+(q-s) r_{t}\left(x^{\prime}\right)\right] \frac{1}{q}\right) & x \in C, q>s .\end{cases}
$$

It is clear that $J_{t}$ is defined and continuous outside $C$. However, we must again check that $J_{t}$ is defined on $C$. For any $x \in C$, we have $x \in B \cap W$ and $x^{\prime} \in B \cap A$. Thus, $R_{t}(x)$ and $r_{t}\left(x^{\prime}\right)$ are defined. Next, since $s \leq \Omega \leq \beta$, the expression $\left\langle r_{t}\left(x^{\prime}\right), R_{t}(x)\right\rangle(1 / \beta)=[(\beta-$ $\left.s) r_{t}\left(x^{\prime}\right)+s R_{t}(x)\right](1 / \beta)$ represents a point lying on the straight path in $\mathbb{R}^{n}$ between $r_{t}\left(x^{\prime}\right)$ and $R_{t}(x)$. Now for each $t \in I$ the distance from $R_{t}(x)$ to $r_{t}\left(x^{\prime}\right)$ satisfies the following inequality:

$$
\begin{aligned}
d\left(R_{t}(x), r_{t}\left(x^{\prime}\right)\right) & \leq d\left(R_{t}(x), x\right)+d\left(x, x^{\prime}\right)+d\left(x^{\prime}, r_{t}\left(x^{\prime}\right)\right) \\
& =d\left(R_{t}(x), R_{0}(x)\right)+d\left(\psi_{0}(x), \psi_{1}(x)\right)+d\left(r_{0}\left(x^{\prime}\right), r_{t}\left(x^{\prime}\right)\right) \\
& <\varepsilon_{1}+\varepsilon_{3}+\varepsilon_{2}<\varepsilon .
\end{aligned}
$$

Since $\varepsilon=\min \left(d\left(X, V^{c}\right), d\left(\Phi, U^{c}\right)\right)$ and $R_{t}(x), r_{t}\left(x^{\prime}\right) \in X$, all points on the straight path between $r_{t}\left(x^{\prime}\right)$ and $R_{t}(x)$ must lie in $V$. Therefore, the expression

$$
\rho_{t}\left(\left\langle r_{t}\left(x^{\prime}\right), R_{t}(x)\right\rangle \frac{1}{\beta}\right)=\rho_{t}\left(\left[(\beta-s) r_{t}\left(x^{\prime}\right)+s R_{t}(x)\right] \frac{1}{\beta}\right)
$$

is defined for $x \in C$. Moreover, as a composition of continuous functions, the expression is continuous. Therefore, $J_{t}$ is defined and continuous for $x \in C$ with $q=s$.

For $x \in C$ with $q<s$ or $q>s$, a similar argument to that of the proof above for $H_{t}$ shows that $J_{t}(x)$ is defined and continuous. It is straightforward to check that $J_{t}$ is continuous on $X$ and that $J_{t}$ is a homotopy of pairs. 
We denote the third and final homotopy in the construction by $K_{t}:(B, B \cap A) \rightarrow$ $\left(\Phi, \Phi_{A}\right)$, where

$$
K_{t}(x)= \begin{cases}r_{1}(x) & x \in B \cap A, \\ R_{1}(x) & x \in(B-B \cap A)-C, \\ \varphi_{t}\left(\left\langle r_{1}\left(x^{\prime}\right), R_{1}(x)\right\rangle \frac{1}{\beta}\right) & x \in C, q=s, \\ \varphi_{t}\left(\left[\left\langle r_{1}\left(x^{\prime}\right), R_{1}(x)\right\rangle \frac{q}{\beta}+(s-q) R_{1}(x)\right] \frac{1}{s}\right) & x \in C, q<s, \\ \varphi_{t}\left(\left[\left\langle r_{1}\left(x^{\prime}\right), R_{1}(x)\right\rangle \frac{s}{\beta}+(q-s) r_{1}\left(x^{\prime}\right)\right] \frac{1}{q}\right) & x \in C, q>s .\end{cases}
$$

We must again check that our homotopy is defined on $C$. For $x \in C$, all points in the expression $\left\langle r_{1}\left(x^{\prime}\right), R_{1}(x)\right\rangle(1 / \beta)=\left[(\beta-s) r_{1}\left(x^{\prime}\right)+s R_{1}(x)\right](1 / \beta)$ lie on the straight path between $r_{1}\left(x^{\prime}\right)$ and $R_{1}(x)$. From (5.14), the length of this path must be less than $\varepsilon$. Since $\varepsilon=\min \left(d\left(X, V^{c}\right), d\left(\Phi, U^{c}\right)\right)$ and $r_{1}\left(x^{\prime}\right), R_{1}(x) \in \Phi$, all points on this path must lie in $U$. Therefore, $\varphi_{t}\left(\left\langle r_{1}\left(x^{\prime}\right), R_{1}(x)\right\rangle(1 / \beta)\right)=\varphi_{t}\left(\left[(\beta-s) r_{1}\left(x^{\prime}\right)+s R_{1}(x)\right](1 / \beta)\right)$ is defined. The proof that $K_{t}(x)$ is defined for $x \in C$ with $q<s$ or $q>s$ is similar to that of $H_{t}$ and $J_{t}$, and hence omitted. It is straightforward to check that $K_{t}$ is continuous on $X$ and that $K_{t}$ is a homotopy of pairs.

Finally, we define our strong deformation retraction of pairs as $\mathscr{R}_{t}:(B, B \cap A) \rightarrow$ $\left(\Phi, \Phi_{A}\right)$, where

$$
\mathscr{R}_{t}(x)= \begin{cases}H_{3 t}(x) & 0 \leq t \leq 1 / 3 \\ J_{3 t-1}(x) & 1 / 3 \leq t \leq 2 / 3 \\ K_{3 t-2}(x) & 2 / 3 \leq t \leq 1\end{cases}
$$

One can check that for all $x \in B, \mathscr{R}_{1 / 3}(x)=H_{1}(x)=J_{0}(x)$ and $\mathscr{R}_{2 / 3}(x)=J_{1}(x)=K_{0}(x)$. It remains to show that $\mathscr{R}_{t}$ is indeed a strong deformation retraction of pairs. In other words, we must check that $\mathscr{R}_{0}=i d_{B}, \mathscr{R}_{1}(B) \subseteq \Phi, \mathscr{R}_{1}(B \cap A) \subseteq \Phi_{A}$, and for all $t \in I$, $\left.\mathscr{R}_{t}\right|_{\Phi}=i d_{\Phi}$ and $\left.\mathscr{R}_{t}\right|_{\Phi_{A}}=i d_{\Phi_{A}}$. By the construction of $\mathscr{R}_{t}$, it is clear that $\mathscr{R}_{0}=H_{0}(x)=i d_{B}$, $\mathscr{R}_{1}(B \cap A) \subseteq \Phi_{A}$, and $\left.\mathscr{R}_{t}\right|_{\Phi_{A}}=i d_{\Phi_{A}}$.

To see that $\mathscr{R}_{1}(B) \subseteq \Phi$, observe that $\mathscr{R}_{1}(x)=J_{1}(x)$ for all $x$. It is clear that $J_{1}(x) \in \Phi$ for all $x \in B-C$. For $x \in C$, the point $J_{1}(x)$ is obtained by evaluating $\varphi_{1}$ at some point in $U$. Since $\varphi_{t}: U \rightarrow \Phi$ is a strong deformation retraction, $\varphi_{1}(U) \subseteq \Phi$. Therefore, $J_{1}(x) \in \Phi$ for all $x \in C$, and hence for all $x \in B$.

Finally, to see that $\left.\mathscr{R}_{t}\right|_{\Phi}=i d_{\Phi}$, choose any $x \in \Phi$. If $x \in \Phi \cap B-C$ then $\mathscr{R}_{t}(x)=$ $R_{t}(x)=x$. If $x \in C \cap \Phi$, then $d(x, \Phi)=0=q<s$, whence $H_{t}(x)=\rho_{1-t}(x)=x, K_{t}(x)=$ $\rho_{t}\left(R_{t}(x)\right)=x$, and $J_{t}(x)=\rho_{t}\left(R_{1}(x)\right)=x$. Therefore, $\left.\mathscr{R}_{t}\right|_{\Phi}=i d_{\Phi}$, which completes the proof.

We now use Ng's results with Lemma 5.1 and Theorem 5.2 to show that the hypotheses and results from Theorem 4.7 hold for all locally contractible closed subsets of $X$. 
Theorem 5.3. Let $f:(X, A) \rightarrow(X, A)$ be a map of a compact polyhedral pair in which $X$ and $A$ have no local cutpoints. Suppose $\left(\Phi, \Phi_{A}\right)$ is a subset pair in which $\Phi, \Phi_{A}$, and $\Phi \cap Z$ are closed, locally contractible subsets of $X$ such that

(1) $A-\Phi_{A}$ and all components of $X-(A \cup \Phi)$ are not 2-manifolds,

(2) $f$ satisfies $\left(\mathrm{C1}^{\prime}\right)$ and $\left(\mathrm{C} 2^{\prime}\right)$ for $\Phi$,

(3) $\Phi_{A}$ can be by-passed in $A, \Phi \cap Z$ can be by-passed in $Z$, and $\partial A$ can be by-passed in $Z$. Then for every closed subset $\Gamma$ of $\Phi$ that has nonempty intersection with every component of $\Phi_{A}$ and every component of $\Phi \cap Z$, there exists a map $g \sim_{A} f$ with Fix $g=\Gamma$.

Proof. If $\Phi=\varnothing$, then this theorem reduces to a special case of [10, Lemma 3.1]. Thus, we may assume $\Phi \neq \varnothing$. Let $K$ be a triangulation of $X=|K|$. By [2, Proposition 8.12, page 83], both $\Phi$ and $\Phi_{A}$ are finite-dimensional ANR's.

From Theorem 5.2, there exists a subset $B$ of $X$ such that $\Phi \subseteq B$ and the pair $(B, B \cap A)$ strong deformation retracts onto the pair $\left(\Phi, \Phi_{A}\right)$. Lemma 3.1 guarantees that we can find a star cover $\mathrm{St}_{K^{\prime}}(\Phi)$ of $\Phi$ with respect to a sufficiently small subdivision $K^{\prime}$ of $K$ such that $\mathrm{St}_{K^{\prime}}(\Phi) \subseteq B$ and the sets $A-\left(\operatorname{St}_{K^{\prime}}(\Phi) \cap A\right)$ and $X-\left(A \cup \mathrm{St}_{K^{\prime}}(\Phi)\right)$ are not 2-manifolds. It follows from Lemma 5.1 , by restricting the retraction, that $f$ satisfies $\left(\mathrm{C}^{\prime}\right)$ and $\left(\mathrm{C}^{\prime}\right)$ for $\operatorname{St}_{K^{\prime}}(\Phi)$.

By assumption, $\Phi_{A}$ can be by-passed in $A$. Since $A$ is a polyhedron, Theorem 2.2 shows that $\Phi_{A}$ can be neighborhood by-passed in $X$. Likewise, $\Phi \cap Z$ can be neighborhood bypassed in $Z$. Therefore $K^{\prime}$ may be chosen with mesh small enough so that $\operatorname{St}_{K^{\prime}}(\Phi) \cap A$ can be by-passed in $A$ and $\operatorname{St}_{K^{\prime}}(\Phi) \cap Z$ can be by-passed in $Z$. Then $\operatorname{St}_{K^{\prime}}(\Phi) \cap(X-A)$ can also be by-passed in $Z$. Thus any path with endpoints in $X-A$ is homotopic to a path in $Z=\mathrm{cl}(X-A)$. But $\partial A$ can also be by-passed in $Z$, implying that such a path must be homotopic to a path in $X-A$. Therefore $\operatorname{St}_{K^{\prime}}(\Phi) \cap(X-A)$ can be by-passed in $X-A$.

Now by the construction of star covers, each component of $\operatorname{St}_{K^{\prime}}(\Phi)$ contains a component of $\Phi$ and every component of $\Phi$ is contained in a component of $\operatorname{St}_{K^{\prime}}(\Phi)$. Choose any closed subset $\Gamma$ of $\Phi$, having nonempty intersection with every component of $\Phi_{A}$ and every component of $\Phi \cap Z$. Then $\Gamma$ also has nonempty intersection with every component of $\operatorname{St}_{K^{\prime}}(\Phi) \cap A$ and with every component of $\operatorname{St}_{K^{\prime}}(\Phi) \cap Z$. Finally, since $K^{\prime}$ is a triangulation of both $X$ and $A$, the set $\operatorname{St}_{K^{\prime}}(\Phi) \cap A$ is a subpolyhedron of $A$ and thus itself a polyhedron. Therefore, the result follows from Theorem 4.7.

Corollary 5.4. Let $f:(X, A) \rightarrow(X, A)$ be a map of a compact polyhedral pair. Suppose $\left(\Phi, \Phi_{A}\right)$ is a subset pair in which both $\Phi$ and $\Phi_{A}$ are closed, locally contractible subsets of $X$ such that

(1) $A-\Phi_{A}$ and all components of $X-(A \cup \Phi)$ are not 2-manifolds,

(2) $f$ satisfies $\left(\mathrm{C1}^{\prime}\right)$ and $\left(\mathrm{C} 2^{\prime}\right)$ for $\Phi$,

(3) $\Phi_{A}$ can be by-passed in $A, \Phi \cap Z$ can be by-passed in $Z$, and $\partial A$ can be by-passed in $Z$. Then there exists a map $g \sim_{A} f$ with Fix $g=\Phi$.

Notice that in the statement of Theorem 5.3, we require both $\Phi$ and $\Phi_{A}$ to be locally contractible. If $\Phi$ is locally contractible, it does not necessarily follow that $\Phi_{A}$ is locally contractible. For instance, the intersection of the subset $\Phi$ in Example 3.6 with the curve $y=1 / x^{2}(x \geq 0)$ is an infinite sequence of discrete points converging to $(0,0)$ and therefore not locally contractible at the origin. 
We conclude this paper with an example of a map $f:(X, A) \rightarrow(X, A)$ of a polyhedral pair, having a locally contractible subset pair $\left(\Phi, \Phi_{A}\right)$, for which there exists $g \sim_{A} f$ with Fix $g=\Phi$.

Example 5.5. Consider the subset $\Phi$ in Example 3.6. Let $A=\Phi \cap R$ where $R$ denotes the closed rectangle

$$
R=\left\{(x, y) \in \mathbb{R}^{2} \mid-2 \leq x \leq 0,-\varepsilon \leq y \leq \varepsilon\right\},
$$

for any positive real number $\varepsilon<1 / 2$. Then $\Phi_{A}=A$, vacuously implying that $A-\Phi_{A}$ is not a 2-manifold and that $\Phi_{A}$ can be by-passed in $A$. As we saw in Example 3.6, the only component of $X-(A \cup \Phi)=X-\Phi$ is not a 2 -manifold. It is also easy to check that $\Phi \cap Z$ can be by-passed in $Z$ and that $\partial A$ can be by-passed in $Z$.

Let $f:(X, A) \rightarrow(X, A)$ be the map flipping $X$ over the $x$-axis. That is, $f(x, y)=(x,-y)$, as in Example 3.6. It remains to show that $f$ satisfies $\left(\mathrm{Cl}^{\prime}\right)$ and $\left(\mathrm{C}^{\prime}\right)$ for $\Phi$.

To see that $f$ satisfies $\left(\mathrm{C1}^{\prime}\right)$, recall from Example 3.6 that $\Phi$ is homotopy equivalent to $\mathbb{F}_{1} \cup \mathbb{F}_{2}$, the union of the two fixed point classes of $f$. Likewise, $\Phi_{A}$ is homotopy equivalent to $\mathbb{F}_{1}$. As $f\left(\Phi_{A}\right) \subseteq \Phi_{A}$, the homotopy $H_{\Phi}$ from (C1) in Example 3.6 also maps $\Phi_{A}$ to itself. In other words, we can write $H_{\Phi}: \Phi \times I \rightarrow X$ as the homotopy of pairs $\tilde{H}:\left(\Phi, \Phi_{A}\right) \times I \rightarrow$ $(X, A)$.

Next we must show that $f_{A}$ satisfies $(\mathrm{C} 1)$ and $(\mathrm{C} 2)$ for $\Phi_{A}$. The restriction $H_{\Phi_{A}}=$ $\left.\tilde{H}\right|_{\Phi_{A} \times I}$ provides the necessary homotopy from $f_{A}$ to the inclusion $\left.i\right|_{A}$, proving (C1). To see $(\mathrm{C} 2)$, observe that $f_{A}$ has only one essential fixed point class $\mathbb{F}=\mathbb{F}_{1} \cap A$. By choosing the path $\alpha: I \rightarrow Z$ to be a constant path at any point in $\mathbb{E}$, we see that $f_{A}$ satisfies (C2) for $\Phi_{A}$.

Finally, to check that $f$ satisfies $\left(\mathrm{C} 2^{\prime}\right)$, observe that both essential classes $\mathbb{F}_{1}$ and $\mathbb{F}_{2}$ intersect $Z$. For $\mathbb{F}_{1}$, choose the path $\alpha: I \rightarrow Z$ to be the constant path at the origin. As the origin lies in both $Z$ and $\Phi$, the path $\alpha$ fulfills the requirements of $\left(\mathrm{C}^{\prime}\right)$. Similarly for $\mathbb{F}_{2}$, we can choose $\alpha: I \rightarrow Z$ to be the constant path at the point $(8,0)$.

Thus $f$ satisfies all the hypotheses of Theorem 5.3 , implying that for every closed subset $\Gamma$ of $\Phi$ that has nonempty intersection with $\Phi_{A}$ and with both components of $\Phi \cap Z$, there exists a map $g \sim_{A} f$ with Fix $g=\Gamma$. In particular, there exists a map homotopic to $f$ via a homotopy of pairs whose fixed point set is $\Phi$ itself.

\section{Acknowledgment}

I would like to thank Jerzy Dydak of the University of Tennessee, who provided the proof of Theorem 2.2.

\section{References}

[1] R. F. Brown, The Lefschetz Fixed Point Theorem, Scott, Foresman, Illinois, 1971.

[2] A. Dold, Lectures on Algebraic Topology, Die Grundlehren der mathematischen Wissenschaften, vol. 200, Springer, New York, 1972.

[3] J. Dydak, private communication, 2003.

[4] S.-T. Hu, Theory of Retracts, Wayne State University Press, Michigan, 1965. 
[5] J. Jezierski, A modification of the relative Nielsen number of H. Schirmer, Topology and Its Applications 62 (1995), no. 1, 45-63.

[6] B. J. Jiang, On the least number of fixed points, American Journal of Mathematics 102 (1980), no. $4,749-763$.

[7] Lectures on Nielsen Fixed Point Theory, Contemporary Mathematics, vol. 14, American Mathematical Society, Rhode Island, 1983.

[8] C. W. Ng, Fixed point sets of maps of pairs, Ph.D. thesis, University of California at Los Angeles, California, 1995.

[9] H. Schirmer, A relative Nielsen number, Pacific Journal of Mathematics 122 (1986), no. 2, 459473.

[10] _ Fixed point sets in a prescribed homotopy class, Topology and Its Applications 37 (1990), no. $2,153-162$.

[11] _ A survey of relative Nielsen fixed point theory, Nielsen Theory and Dynamical Systems (South Hadley, MA, 1992), Contemp. Math., vol. 152, American Mathematical Society, Rhode Island, 1993, pp. 291-309.

[12] P. Strantzalos, Eine charakterisierung der fixpunktmengen bei selbstabbildungen kompakter mannigfaltigkeiten aus einer homotopieklasse, Bulletin de l'Académie Polonaise des Sciences. Série des Sciences Mathématiques, Astronomiques et Physiques 25 (1977), no. 8, 787-793.

[13] X. Z. Zhao, Estimation of the number of fixed points on the complement, Topology and Its Applications 37 (1990), no. 3, 257-265.

Christina L. Soderlund: Department of Mathematics, California Lutheran University, 60 West Olsen Road 3750, Thousand Oaks, CA 91360-2700, USA

E-mail address: csoderlu@callutheran.edu 